\title{
Synthesis and biological evaluation of 4-thiazolidinone derivatives as potential antimycobacterial agents
}

\author{
Tumul Srivastava, ${ }^{\text {a }}$ Anil K. Gaikwad, ${ }^{\text {b Wahajul Haq, }}$ Sudhir Sinha, ${ }^{\text {b }}$ and Setu B. Katti ${ }^{* a}$ \\ ${ }^{a}$ Medicinal \& Process Chemistry Division, ${ }^{b}$ Drug Target Discovery \& Development Division, \\ Central Drug Research Institute, Lucknow 226001, India \\ E-mail: setu_katti@yahoo.com
}

\section{Dedicated to Dr. Nitya Anand on the occasion of his $\mathbf{8 0}^{\text {th }}$ birthday}

(received 04 Nov 04; accepted 08 Mar 05; published on the web 16 Mar 05)

\begin{abstract}
In a one pot procedure, amines 1a-c, cyclic ketones 3a-f and mercapto acids 2a-c were converted into 1-thia-4-azaspiro[4.n]alkan-3-ones $(\mathrm{n}=4-7) \mathbf{4 a - e , ~ 8 a - e , ~ 9 a ~ a n d ~ 1 - t h i a - 4 , 8 - d i a z a - ~}$ spiro[4.5]decan-3-one $\mathbf{9 b}$. The 4-thiazolidinone moiety of $\mathbf{4 b}$ and the piperidine ring of $\mathbf{9 b}$ were subsequently derivatized furnishing products 5-7 and 10, 12a-d, respectively. The products were evaluated as potential antimycobacterial agents, ten compounds were found active at $25 \mu \mathrm{g} / \mathrm{mL}$ concentration; with the most active compound 12a showing more than $90 \%$ inhibition.
\end{abstract}

Keywords: 4-Thiazolidinones, microplate Alamar blue assay (MABA), antimycobacterial

\section{Introduction}

Tuberculosis (TB) remains the major cause of death all over the world. Emergence of multi drug resistant tuberculosis (MDR-TB) makes the conditions most alarming. Up to $4 \%$ of all TB cases worldwide are resistant to more than one antitubercular drug because of incomplete or partial therapy. ${ }^{1}$ Therefore, there is an urgent demand for a new class of antitubercular agents with a different mode of action. A de novo structure-based design has demonstrated that the 4-thiazolidinone scaffold inhibits an enzyme RmlC, which is an essential component for the biosynthesis of dTDP-rhamnose ${ }^{2}$. This prompted us to communicate our findings in this manuscript. 


\section{Results and Discussion}

\section{Chemistry}

In a one-pot procedure, 1-thia-4-azaspiro[4.5]decan-3-ones 4a-e were obtained in the course of a three component reaction with amines 1a-c, cyclohexanone (3a) and mercapto acids 2a,b using dicyclohexylcarbodiimide (DCC) as dehydrating agent in dry THF within one hour (Scheme1). ${ }^{3}$ Oxidation of compound $\mathbf{4 b}$ with Oxone reagent under different conditions gave sulfoxide 5 and sulfone 6. The reaction was carried out at -5 to $-10{ }^{\circ} \mathrm{C}$ in methanol/water (1:1): After one hour sulfoxide 5 was isolated; continuing the reaction at room temperature for additional two hours furnished sulfoxide 6. By heating with Lawesson's reagent in toluene at reflux for two hours the thiazolidinone $\mathbf{4 b}$ was converted into the corresponding thione 7 (Scheme 1).

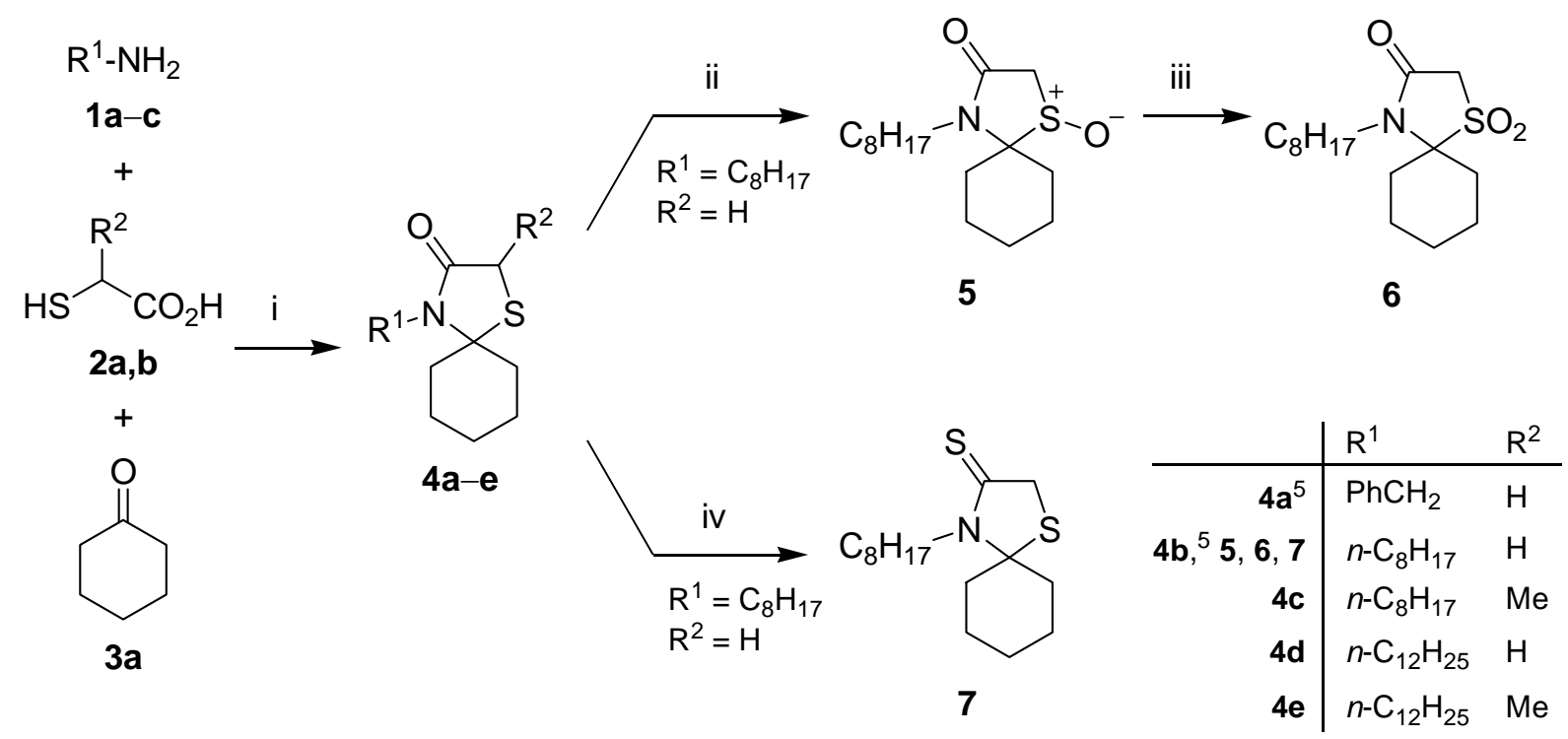

Scheme 1. Reagents and conditions: i. DDC, THF, r.t., 1 h. ii. Oxone, $\mathrm{MeOH} / \mathrm{H}_{2} \mathrm{O}(1: 1),-5$ to $-10{ }^{\circ} \mathrm{C}, 1$ h. iii. Oxone, $\mathrm{MeOH} / \mathrm{H}_{2} \mathrm{O}$ (1:1), r.t., 2 h. iv. Lawesson's reagent, toluene, reflux, 2 h.

When the monoacids 2a,b were replaced by mercaptosuccinic acid 2c an interference of the additional carboxyl group with DCC was anticipated, ${ }^{3}$ and therefore, a slightly different strategy was adopted . ${ }^{4}$ In this protocol, the synthesis of 1-thia-4-azaspiro[4.5]decan-3-ones 8a-e (Scheme 2), 8-tert-butyl-1-thia-4-azaspiro[4.5]decan-3-one 9a and N-Boc-protected 1-thia-4,8diazaspiro[4.5]decan-3-one 9b (Scheme 3) was achieved by refluxing a mixture of amines 1b,c, mercaptosuccinic acid 2c and carbonyl compounds 3a-f in toluene for 10-12 hours.

Removal of the Boc group in 1-thia-4,8-diazaspiro[4.5]decan-3-one 9 b with $20 \%$ hydrogen chloride/dioxane solution afforded the hydrochloride 10 (Scheme 3). 


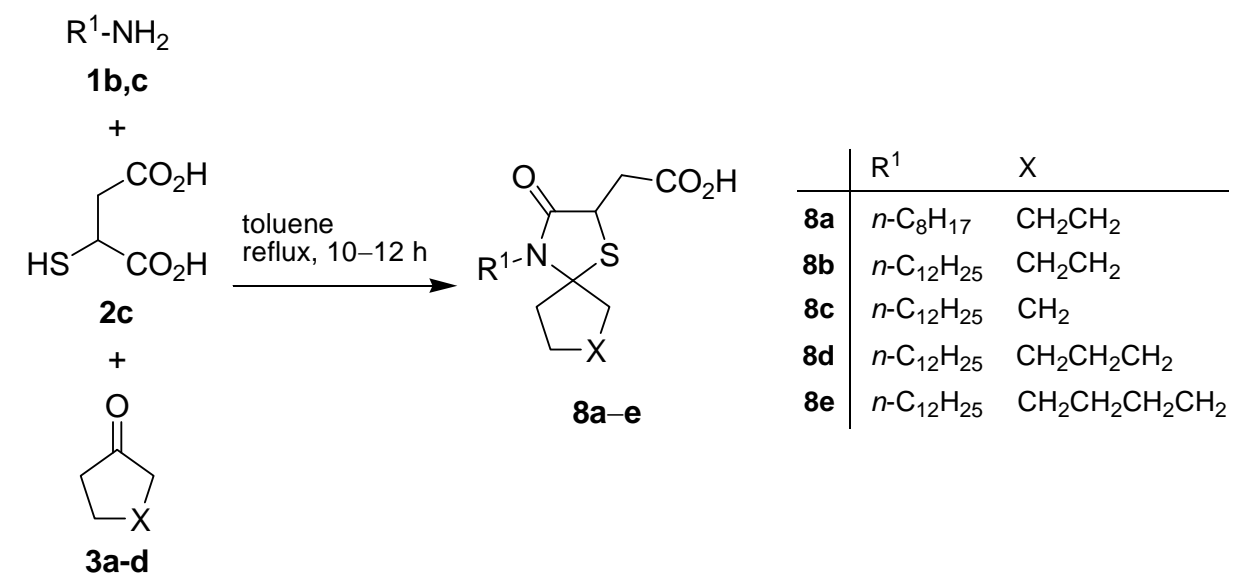

\section{Scheme 2}
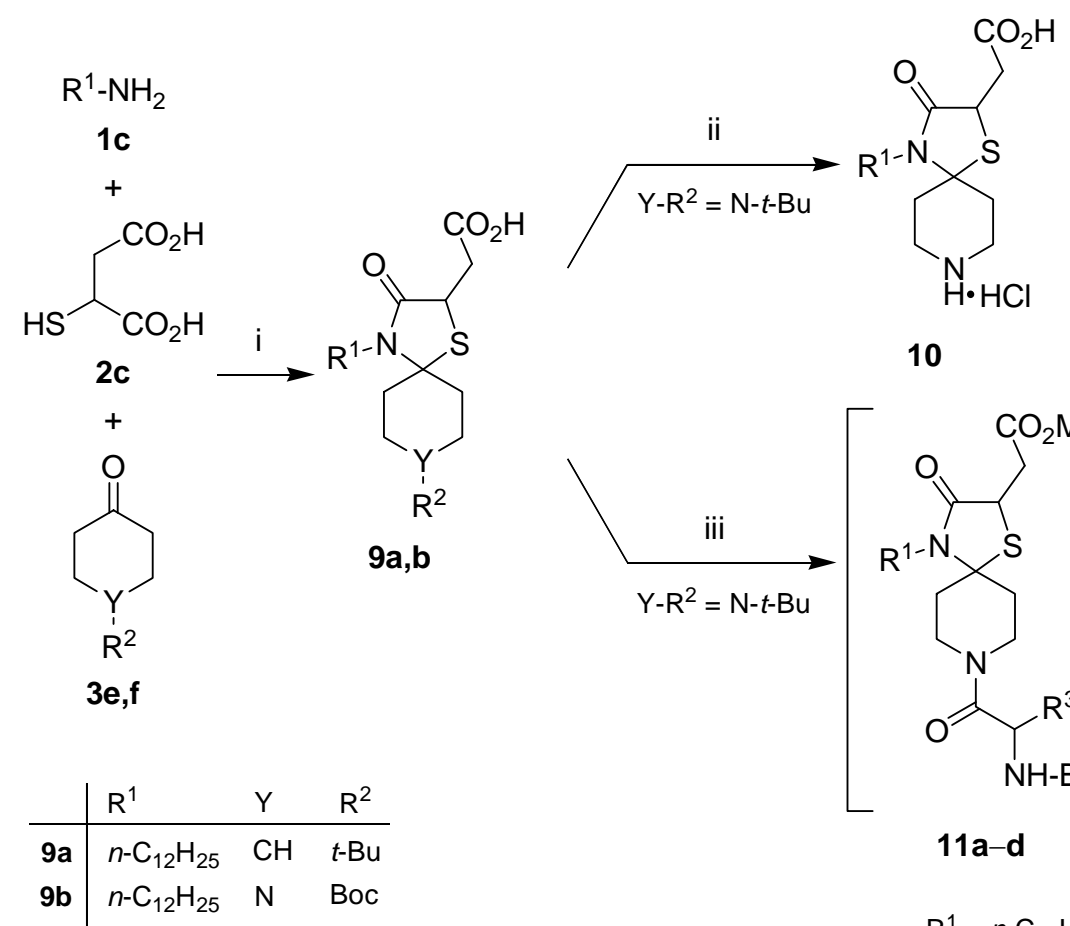

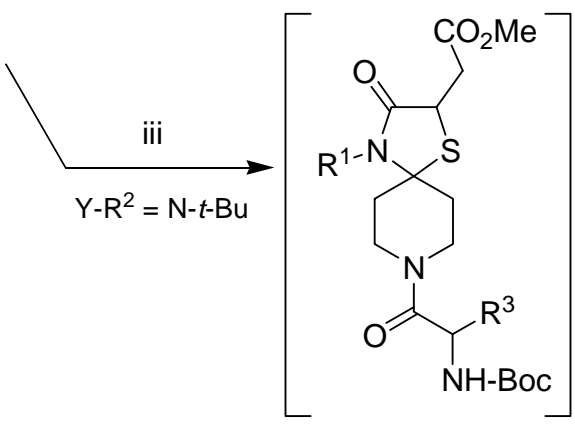

$11 a-d$

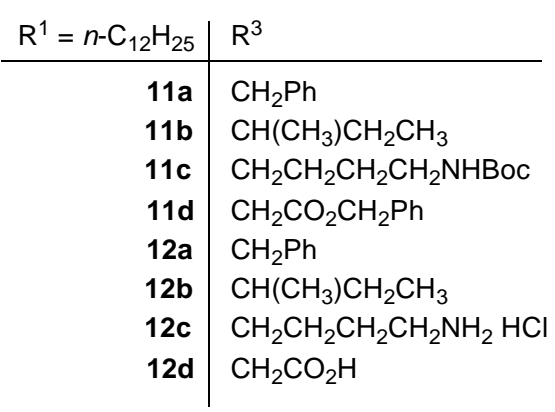

Scheme 3. Reagents and conditions: i. Toluene, reflux, $10-12$ h. ii. $\mathrm{CH}_{2} \mathrm{~N}_{2} /$ ether, $\mathrm{MeOH},-5{ }^{\circ} \mathrm{C}$, $10 \mathrm{~min} ; 20 \% \mathrm{HCl} /$ doxane, r.t., 1 h; $\mathrm{Boc}-\mathrm{NHCH}\left(\mathrm{R}^{3}\right) \mathrm{CO}_{2} \mathrm{H}$, HBTU, DIEA, THF, r.t., 1 h. iv. $\mathrm{LiOH}, \mathrm{THF} / \mathrm{MeOH} / \mathrm{H}_{2} \mathrm{O}$ (7:2:1), 1 h; for 11c: $\mathrm{Pd} / \mathrm{C}-\mathrm{H}_{2}, \mathrm{MeOH}$, r.t., 4 h. 
Compound 9b was treated with an ether solution of diazomethane, and after removal of the ether solvent the corresponding crude methyl ester derivative was obtained, which was subsequently treated with $20 \%$ hydrogen chloride in dioxane solution to remove the Boc group. Evaporation of reaction mixture to dryness gave the crude amine which turned crystalline with diethyl ether. This product was coupled with Boc-protected amino acids using 2-(1Hbenzotriazol-1-yl)-1,1,3,3-tetramethyluronium hexafluorophosphate (HBTU) and diisopropylethyl amine (DIEA) in dry THF at room temperature to furnish coupling products 11a-d. After isolation and purification, the coupling products 11a-c were treated with lithium hydroxide in $\mathrm{THF} / \mathrm{methanol} /$ water $(7: 2: 1)$ for one hour and the products obtained after isolation were treated separately with $20 \%$ hydrogen chloride in dioxane solution to provide products 12a-c. Compound 11d was treated with lithium hydroxide in THF/methanol/water $(7: 2: 1)$, and the isolated product was subjected to catalytic hydrogenation over $\mathrm{Pd} / \mathrm{C}$. In the hydrogenation process the O-benzyl group was removed from the aspartate moiety; the crude product obtained after work up was treated with $20 \%$ hydrogen chloride in dioxane solution to furnish the hydrochloride salt 12d (Scheme 3).

\section{Biological activity}

The above synthesized products were screened against $M$. tuberculosis using Microplate Alamar Blue Assay (MABA) assay ${ }^{5}$ on High Throughput Screening (HTS) machine at $25 \mu \mathrm{g} / \mathrm{mL}$ and lower concentrations using $M$. tuberculosis H37Ra as a surrogate for the virulent H37Rv strain. The results are shown in table 1 . The results of MABA have been found comparable to standard BACTEC 460 system based assay. The standard antitubercular drugs Rifamycin, Isoniazid, paminosalicylic acid, Ethambutol and Ethionamide (MIC range 3-0.3 $\mu \mathrm{g} / \mathrm{mL}$ ) were taken as positive controls. We have also done cytotoxicity analysis of the above synthesized compounds, using neutral red uptake by using Vero-C-1008 cell line at various concentrations $(6.25 \mu \mathrm{g} / \mathrm{mL}$ to $50 \mu \mathrm{g} / \mathrm{mL}$ ), none of them were found toxic. Hence the activities of the above synthesized compounds were not due to cytotoxicity of the compounds.

Table 1. Percentage inhibition at $25 \mu \mathrm{g} / \mathrm{mL}$ concentration

\begin{tabular}{cccccc}
\hline Compound & Activity (\%) & Compound & Activity (\%) & Compoundd & Activity (\%) \\
\hline 4a & 8 & $\mathbf{7}$ & 7 & $\mathbf{9 b}$ & 96 \\
4b & 96 & $\mathbf{8 a}$ & 8 & $\mathbf{1 0}$ & 3 \\
4c & 87 & $\mathbf{8 b}$ & 97 & $\mathbf{1 2 a} *$ & 97 \\
$\mathbf{4 d}$ & 5 & $\mathbf{8 c}$ & 97 & $\mathbf{1 2 b}$ & 97 \\
4e & 2 & $\mathbf{8 d}$ & 96 & $\mathbf{1 2 c}$ & 96 \\
$\mathbf{5}$ & 5 & $\mathbf{8 e}$ & 96 & $\mathbf{1 2 d}$ & 2 \\
$\mathbf{6}$ & 81 & $\mathbf{9 a}$ & 96 & & \\
\hline
\end{tabular}

*Compound showed $94 \%$ inhibition at $12.5 \mu \mathrm{g} / \mathrm{mL}$. 
During the preliminary screening five compounds $4 a-\mathbf{e}$ were tested (Table 1) at $25 \mu \mathrm{g} / \mathrm{mL}$ concentration for their antimycobacterial activity. One of the compounds $\mathbf{4 b}$ have exhibited $96 \%$ inhibition at this concentration while other compounds 4a and 4c-e exhibited less than $90 \%$ inhibition at the same concentration. We have taken $\mathbf{4 b}$ as a lead molecule and subsequent structural modifications were carried out on this compound. As a first step towards lead optimization sulfur was oxidized to the corresponding sulfoxide 5 and sulfone $\mathbf{6}$ however, both of these modifications were resulted in a substantial decrease in activity. The next structural modification made was to convert carbonyl moiety to the corresponding thione 7 but this change were also resulted in a substantial loss of biological activity. Therefore, these centers were not modified in the subsequent studies.

Compound $\mathbf{8 b}$ has shown $97 \%$ inhibition at $25 \mu \mathrm{g} / \mathrm{mL}$ (Table 1). Therefore this compound was chosen for further studies. In order to optimize the carbonyl component, five compounds 8ce and 9a,b were synthesized and investigated. The results of the antimycobacterial activity are quite interesting because all of these compounds have shown inhibition above $90 \%$ at $25 \mu \mathrm{g} / \mathrm{mL}$ (Table 1). On the other hand, in secondary screening at $12.5 \mu \mathrm{g} / \mathrm{mL}$ concentration these compounds were found to have decreased antimycobacterial activity. Compound 9b was selected for further studies as it has a Boc-protected amino group, which opened an area for further modification at this point. Unprotected 10 was found to have decreased antimycobacterial activity. Furthermore, compounds 12a-d were investigated: 12a-c have shown more than 95\% inhibition at $25 \mu \mathrm{g} / \mathrm{mL}$ concentration and more interestingly, compound 12a has shown 94\% inhibition at $12.5 \mu \mathrm{g} / \mathrm{mL}$ concentration. Although we have not been able to substantially enhance the activity of 4-thiazolidinones in the present study, the data presented here are encouraging and deserve further investigation.

\section{Conclusions}

In the present study synthesis and antimycobacterial activity of 4-thiazolidinone derivatives have been described. Some of these derivatives (4b, 8b-e, 9a,b, 12a-c) have shown more than 90\% inhibition at $25 \mu \mathrm{g} / \mathrm{mL}$ concentration. Moreover, one compound, 12a has been found to be the most active, it showed more than $90 \%$ inhibition at $12.5 \mu \mathrm{g} / \mathrm{mL}$ concentration. These results confirm the fact that the 4-thiazolidinone skeleton has great potential as antimycobacterial agent. It is thus concluded that 4-thiazolidinones deserve further investigation for the development of more potent and non toxic antitubercular agents for therapeutic use.

\section{Experimental Section}

General Procedures. Melting points (mp) were determined on a Complab melting point apparatus. Thin-layer chromatography (tlc) was performed on readymade silica gel plates 
(Merck); iodine was used as visualizing reagent. Products were purified by column chromatography using silica gel (230-400 mesh). The CHN analyses were carried out on CARLO-ERBA EA1108 elemental analyser. Infrared (IR) spectra were recorded on an FT-IR Perkin-Elmer spectrometer. The ${ }^{1} \mathrm{H}$ and ${ }^{13} \mathrm{C}$ NMR spectra were recorded on a DPX-200 Bruker FT-NMR spectrometer. The chemical shifts are reported with reference to TMS as internal standard. Mass spectra were obtained on a JEOL-SX-102 instrument using fast atom bombardment (FAB positive) technique.

\section{1-Thia-4-azaspiro[4.5]decan-3-ones (4a-e). General procedure A}

A mixture of amine 1a-c $(1.0 \mathrm{mmol})$ and cyclohexanone (3a, $0.20 \mathrm{~mL}, 2.0 \mathrm{mmol})$ was stirred in THF with ice cooling for $5 \mathrm{~min}$, followed by addition of mercaptoacid 2a,b (3.0 mmol). After 5 min DCC (247 mg, $1.2 \mathrm{mmol}$ ) was added to the reaction mixture at $0{ }^{\circ} \mathrm{C}$, and the reaction mixture was stirred for an additional $50 \mathrm{~min}$. at room temperature. Dicyclohexylurea was filtered off, the filtrate was concentrated to dryness under reduced pressure and the residue was taken-up in ethyl acetate. The ethyl acetate layer was washed with $5 \%$ citric acid solution $(2 \mathrm{x})$, with brine $(2 \mathrm{x})$, with $5 \%$ sodium bicarbonate solution $(2 \mathrm{x})$ and finally with brine $(2 \mathrm{x})$. The organic layer was dried over sodium sulfate and evaporated to dryness at reduced pressure. The crude product thus obtained was purified by column chromatography on silica gel with hexane-ethyl acetate as eluent. The purity of the products was checked by tlc using ethyl acetate/hexane (4:6).

The preparation of $\mathbf{4 a}$ and $\mathbf{4 b}$ has been described previously. ${ }^{5}$

2-Methyl-4-octyl-1-thia-4-azaspiro[4.5]decan-3-one (4c). Following General Procedure A, $n$-octylamine $\mathbf{1 b}(0.17 \mathrm{~mL}, 1.0 \mathrm{mmol})$, cyclohexanone 3a $(0.20 \mathrm{~mL}, 2.0 \mathrm{mmol})$ and 2-mercaptopropanoic acid $2 \mathbf{b}(0.27 \mathrm{~mL}, 3.0 \mathrm{mmol})$ gave $4 \mathbf{c}(249 \mathrm{mg}, 84 \%)$ as a gum. $\mathrm{R}_{f} 0.65(40 \%$ ethyl acetate/hexane). IR (neat): $\widetilde{v} 1674.4 \mathrm{~cm}^{-1}(\mathrm{C}=\mathrm{O}) .{ }^{1} \mathrm{H}$ NMR $\left(200 \mathrm{MHz}, \mathrm{CDCl}_{3}\right): \delta 0.84(\mathrm{t}, J=$ $\left.6.48 \mathrm{~Hz}, 3 \mathrm{H}, \mathrm{CH}_{3}\left(\mathrm{CH}_{2}\right)_{7}\right), 1.22-1.92\left(\mathrm{~m}, 25 \mathrm{H}, \mathrm{CH}_{3}\left(\mathrm{CH}_{2}\right)_{6} \mathrm{CH}_{2},\left(\mathrm{CH}_{2}\right)_{5}, \mathrm{CHCH}_{3}\right), 3.16$ (t, J = 7.9 $\mathrm{Hz} 2 \mathrm{H}, \mathrm{NCH}_{2}$ ), 3.74 (q, $J=6.98 \mathrm{~Hz}, 1 \mathrm{H}, \mathrm{CHCH}_{3}$ ). FAB-MS: $m / z$ (\%) 298 (100) [M+H] $]^{+}$. Anal. Calcd for $\mathrm{C}_{17} \mathrm{H}_{31} \mathrm{NOS}$ (297.50): C, 68.63; H, 10.50; N, 4.71. Found: C, 68.74; H, 10.58; N, 4.84.

4-Dodecyl-1-thia-4-aza-spiro[4.5]decan-3-one (4d). Following General Procedure A Laurylamine 1c $(0,23 \mathrm{~mL}, 1.0 \mathrm{mmol})$, cyclohexanone $3 \mathbf{a}(0.20 \mathrm{~mL}, 2.0 \mathrm{mmol})$ and mercaptoacetic acid 2b $(0.21 \mathrm{~mL}, 3.0 \mathrm{mmol})$ gave $4 \mathbf{d}(268 \mathrm{mg}, 79 \%)$ as a gum. $\mathrm{R} f 0.76$ (40\% ethyl acetate/hexane). IR (neat): $\widetilde{v} 1660.7 \mathrm{~cm}^{-1}(\mathrm{C}=\mathrm{O}) .{ }^{1} \mathrm{H} \mathrm{NMR}\left(200 \mathrm{MHz}, \mathrm{CDCl}_{3}\right): \delta 0.88(\mathrm{t}, J=6.74 \mathrm{~Hz}, 3 \mathrm{H}$, $\left.\mathrm{CH}_{3}\left(\mathrm{CH}_{2}\right)_{11}\right), \quad 1.25-1.80$ [m, 30H, $\left.\mathrm{CH}_{3}\left(\mathrm{CH}_{2}\right)_{10} \mathrm{CH}_{2},\left(\mathrm{CH}_{2}\right)_{5}\right], 3.19$ (t, J = 8.09 Hz, 2H, $\left.\mathrm{CH}_{3}\left(\mathrm{CH}_{2}\right)_{10} \mathrm{CH}_{2}\right), 3.48$ (s, 2H, 3-CH ). FAB-MS: m/z (\%) 340 (100) [M+H] $]^{+}$. Anal. Calcd for $\mathrm{C}_{20} \mathrm{H}_{37} \mathrm{NOS}$ (339.58): C, 70.74; H, 10.98; N, 4.12. Found: C, 70.79; H, 11.10; N, 4.15.

4-Dodecyl-2-methyl-1-thia-4-aza-spiro[4.5]decan-3-one (4e). Following General Procedure A laurylamine 1c $(0.23 \mathrm{~mL}, 1.0 \mathrm{mmol})$, cyclohexanone $3 \mathbf{a}(0.20 \mathrm{~mL}, 2.0 \mathrm{mmol})$ and 2mercaptopropanoic acid $\mathbf{2 b}(0.27 \mathrm{~mL}, 3.0 \mathrm{mmol})$ gave $4 \mathbf{c}(264 \mathrm{mg}, 75 \%)$ as a gum. $\mathrm{R} f 0.72(40 \%$ ethyl acetate/hexane). IR (neat): $\widetilde{v} 1683.5 \mathrm{~cm}^{-1}(\mathrm{C}=\mathrm{O}) .{ }^{1} \mathrm{H} \mathrm{NMR}\left(200 \mathrm{MHz}, \mathrm{CDCl}_{3}\right): \delta 0.88(\mathrm{t}, J$ $\left.=6.73 \mathrm{~Hz}, 3 \mathrm{H}, \mathrm{CH}_{3}\left(\mathrm{CH}_{2}\right)_{11}\right), 1.22-2.30\left[\mathrm{~m}, 33 \mathrm{H}, \mathrm{CH}_{3}\left(\mathrm{CH}_{2}\right)_{10} \mathrm{CH}_{2},\left(\mathrm{CH}_{2}\right)_{5}, \mathrm{CHCH}_{3}\right], 3.37(\mathrm{t}, J=$ $\left.7.94 \mathrm{~Hz}, 2 \mathrm{H}, \mathrm{CH}_{3}\left(\mathrm{CH}_{2}\right)_{10} \mathrm{CH}_{2}\right), 4.12$ (q, $\left.J=7.12 \mathrm{~Hz}, 1 \mathrm{H}, \mathrm{CHCH}_{3}\right)$. FAB-MS: $m / z(\%) 354$ (100) 
$[\mathrm{M}+\mathrm{H}]^{+}$. Anal. Calcd for $\mathrm{C}_{21} \mathrm{H}_{39} \mathrm{NOS}$ (353.61): C, 71.33; H, 11.12; N, 3.96. Found: C, 71.45; H, 11.20; N, 4.10.

4-Octyl-1-thia-4-azaspiro[4.5]decan-3-one 1-oxide (5). A mixture of $4 \mathbf{b}$ (283 $\mathrm{mg}, 1 \mathrm{mmol})$ and Oxone $\left(2 \mathrm{KHSO}_{4} \cdot \mathrm{KHSO}_{4} \cdot \mathrm{K}_{2} \mathrm{SO}_{4} ; 738 \mathrm{mg}, 1.2 \mathrm{mmol}\right)$ in $50 \%$ methanol/water $(30 \mathrm{~mL})$ at -5 to $10{ }^{\circ} \mathrm{C}$ was stirred for $1 \mathrm{~h}$. The reaction mixture was concentrated under reduced pressure to dryness, and the residue was taken-up in ethyl acetate. The ethyl acetate layer was washed with $5 \%$ citric acid solution $(2 \mathrm{x})$, with brine $(2 \mathrm{x})$, with $5 \%$ sodium bicarbonate solution $(2 \mathrm{x})$, and finally with brine $(2 \mathrm{x})$ before drying over sodium sulfate. The filtrate was concentrated under reduced pressure to dryness to give product $5(287 \mathrm{mg}, 96 \%)$ as a white solid; $\mathrm{R}_{f} 0.18$ (40\% ethyl acetate/hexane); mp $68-71{ }^{\circ} \mathrm{C}$. IR: $(\mathrm{KBr}) \widetilde{v} 1596.2 \mathrm{~cm}^{-1}(\mathrm{C}=\mathrm{O}) .{ }^{1} \mathrm{H}$ NMR $\left(200 \mathrm{MHz}, \mathrm{CDCl}_{3}\right): \delta$ : $0.88\left[\mathrm{t}, J=6.69 \mathrm{~Hz}, 3 \mathrm{H}, \mathrm{CH}_{3}\left(\mathrm{CH}_{2}\right)_{7}\right], 1.27-1.93\left[\mathrm{~m}, 22 \mathrm{H}, \mathrm{CH}_{3}\left(\mathrm{CH}_{2}\right)_{6} \mathrm{CH}_{2},\left(\mathrm{CH}_{2}\right)_{5}\right], 3.06(\mathrm{t}, J=$ $\left.8.2 \mathrm{~Hz}, 2 \mathrm{H}, \mathrm{NCH}_{2}\right), 3.44\left(\mathrm{~d}, 1 \mathrm{H}, J=17.34 \mathrm{~Hz}, 2-\mathrm{CH}_{\mathrm{A}}\right), 3.64\left(\mathrm{~d}, 1 \mathrm{H}, J=17.34 \mathrm{~Hz}, 2-\mathrm{CH}_{\mathrm{B}}\right)$. FABMS m/z (\%) $301(100)[\mathrm{M}+\mathrm{H}]^{+}$. Anal. Calcd for $\mathrm{C}_{16} \mathrm{H}_{29} \mathrm{NO}_{2} \mathrm{~S}(299.47)$ : C, 64.17; H, 9.76; N, 4.68. Found: C, 64.30; H, 9.79; N, 4.77.

4-Octyl-1-thia-4-azaspiro[4.5]decan-3-one 1,1-dioxide (6). A mixture of 4b (283 mg, $1 \mathrm{mmol})$ and Oxone $\left(2 \mathrm{KHSO}_{4} \cdot \mathrm{KHSO}_{4} \cdot \mathrm{K}_{2} \mathrm{SO}_{4} ; 1.85 \mathrm{mg}, 3.0 \mathrm{mmol}\right)$ in $50 \% \mathrm{MeOH} / \mathrm{H}_{2} \mathrm{O}(30 \mathrm{~mL})$ was stirred first at -5 to $-10{ }^{\circ} \mathrm{C}$ and then at room temperature for $2 \mathrm{~h}$. The reaction mixture was worked-up as described for 5 to give product $6(299 \mathrm{mg}, 95 \%)$ as a white solid; $\mathrm{R}_{f} 0.65(40 \%$ ethyl acetate/hexane); mp 50-52 ${ }^{\circ} \mathrm{C}$. IR: (KBr) $\widetilde{v} 1691.7 \mathrm{~cm}^{-1}(\mathrm{C}=\mathrm{O}) .{ }^{1} \mathrm{H}$ NMR $(200 \mathrm{MHz}$, $\left.\mathrm{CDCl}_{3}\right): \delta 0.88\left(\mathrm{t}, J=5.51 \mathrm{~Hz}, 3 \mathrm{H}, \mathrm{CH}_{3}\left(\mathrm{CH}_{2}\right)_{7}\right), 1.27-1.94\left[\mathrm{~m}, 22 \mathrm{H}, \mathrm{CH}_{3}\left(\mathrm{CH}_{2}\right)_{6} \mathrm{CH}_{2},\left(\mathrm{CH}_{2}\right)_{5}\right]$, 3.27 (t, $\left.J=8.15 \mathrm{~Hz}, 2 \mathrm{H}, \mathrm{NCH}_{2}\right), 3.76\left(\mathrm{~s}, 2 \mathrm{H}, 2-\mathrm{CH}_{2}\right)$. FAB-MS m/z (\%) 317 (100) $[\mathrm{M}+\mathrm{H}]^{+}$. Anal. Calcd for $\mathrm{C}_{16} \mathrm{H}_{29} \mathrm{NO}_{3} \mathrm{~S}$ (315.47): C, 60.92; H, 9.27; N, 4.44. Found: C, 61.17; H, 9.28; N, 4.30 .

4-Octyl-1-thia-4-azaspiro[4.5]decane-3-thione (7). A mixture of $4 \mathbf{b}$ (283 $\mathrm{mg}, 1 \mathrm{mmol}$ ) and Lawesson's reagent $(404 \mathrm{mg}, 1 \mathrm{mmol})$ in toluene $(50 \mathrm{~mL})$ was heated at reflux for $2 \mathrm{~h}$. The reaction mixture was was worked-up as described for 5 to yield product $7(260 \mathrm{mg}, 87 \%)$ as a gum; $\mathrm{R}_{f} 0.89$ (40\% EtOAc/Hexane). IR: (neat) $\widetilde{v} 1656.8 \mathrm{~cm}^{-1}(\mathrm{C}=\mathrm{O}) .{ }^{1} \mathrm{H}$ NMR $(200 \mathrm{MHz}$, $\left.\mathrm{CDCl}_{3}\right): \delta 0.85\left[\mathrm{t}, J=5.78 \mathrm{~Hz}, 3 \mathrm{H}, \mathrm{CH}_{3}\left(\mathrm{CH}_{2}\right)_{7}\right], 1.28-1.91\left[\mathrm{~m}, 22 \mathrm{H}, \mathrm{CH}_{3}\left(\mathrm{CH}_{2}\right)_{6} \mathrm{CH}_{2},\left(\mathrm{CH}_{2}\right)_{5}\right]$, $3.66\left(\mathrm{t}, J=8.38 \mathrm{~Hz}, 2 \mathrm{H}, \mathrm{NCH}_{2}\right), 4.08\left(\mathrm{~s}, 2 \mathrm{H}, 2-\mathrm{CH}_{2}\right)$. FAB-MS m/z (\%) $300(100)[\mathrm{M}+\mathrm{H}]^{+}$. Anal. Calcd for $\mathrm{C}_{16} \mathrm{H}_{29} \mathrm{NS}_{2}$ (299.54): C, 64.16; H, 9.76; N, 4.68. Found: C, 64.21; H, 9.86; N, 4.75 .

\section{Synthesis of spiro compounds 8a-e and 9a,b. General procedure B}

A mixture of amine $\mathbf{1 b}, \mathbf{c}(1.0 \mathrm{mmol})$, ketone 3a-f $(2.0 \mathrm{mmol})$ and mercaptosuccinic acid 2c $(450 \mathrm{mg}, 3.0 \mathrm{mmol})$ in dry toluene $(20 \mathrm{~mL})$ was heated at reflux for $10-12 \mathrm{~h}$. The reaction mixture was cooled to room temperature, and the solvent was evaporated, the residue was taken up in ethyl acetate, and the resulting solution was washed with saturated sodium chloride solution. The organic phase was dried over sodium sulfate, filtered, the filtrate was concentrated under reduced pressure to dryness, and the crude product was purified by column 
chromatography on silica gel using methanol/dichloromethane as eluent. The purity of the products were checked by by tlc using methanol/dichloromethane (1:9) as solvent.

4-Octyl-3-oxo-1-thia-4-azaspiro[4.5]dec-2-yl)acetic acid (8a). Applying General Procedure B, n-octylamine $\mathbf{1 b}(0.17 \mathrm{~mL}, 1.0 \mathrm{mmol})$, mercaptosuccinic acid 2c (450 $\mathrm{mg}, 3.0 \mathrm{mmol})$ and cyclohexanone 3a $(0.20 \mathrm{~mL}, 2.0 \mathrm{mmol})$ gave $8 \mathrm{a}(266 \mathrm{mg}, 78 \%)$ as a gum. $\mathrm{R}_{f} 0.46(10 \%$ methanol/dichloromethane). IR (neat): $\widetilde{v} 1637.3,1733.4(\mathrm{C}=\mathrm{O}) \mathrm{cm}^{-1}$. ${ }^{1} \mathrm{H}$ NMR $(200 \mathrm{MHz}$, $\left.\mathrm{CDCl}_{3}\right): \delta 0.88\left[\mathrm{t}, J=6.7 \mathrm{~Hz}, 3 \mathrm{H}, \mathrm{CH}_{3}\left(\mathrm{CH}_{2}\right)_{7}\right], 1.22-1.90\left[\mathrm{~m}, 22 \mathrm{H}, \mathrm{CH}_{3}\left(\mathrm{CH}_{2}\right)_{6} \mathrm{CH}_{2},\left(\mathrm{CH}_{2}\right)_{5}\right], 2.67$ $\left(\mathrm{dd}, J=8.96,17.0 \mathrm{~Hz}, 1 \mathrm{H}, \mathrm{CH}_{\mathrm{A}} \mathrm{CO}_{2} \mathrm{H}\right), 3.08-3.35\left(\mathrm{~m}, 3 \mathrm{H}, \mathrm{CH}_{\mathrm{B}} \mathrm{CO}_{2} \mathrm{H}, \mathrm{NCH}_{2}\right), 4.10$ (dd, $J=4.8$, $8.93 \mathrm{~Hz}, 1 \mathrm{H}, 2-\mathrm{CH})$. FAB-MS: m/z (\%) 342 (100) $[\mathrm{M}+\mathrm{H}]^{+}$. Anal. Calcd for $\mathrm{C}_{18} \mathrm{H}_{31} \mathrm{NO}_{3} \mathrm{~S}$ (341.51): C, 63.30; H, 9.15; N, 4.10. Found: C, 63.50; H, 9.20; N, 4.25.

4-Dodecyl-3-oxo-1-thia-4-azaspiro[4.5]dec-2-yl)acetic acid (8b). Applying General Procedure B, laurylamine 1c $(0.23 \mathrm{~mL}, 1.0 \mathrm{mmol})$, mercaptosuccinic acid 2c $(450 \mathrm{mg}, 3.0 \mathrm{mmol})$ and cyclohexanone 3a $(0.20 \mathrm{~mL}, 2.0 \mathrm{mmol})$ gave $\mathbf{8 b}(297 \mathrm{mg}, 75 \%)$ as a white solid; $\mathrm{R}_{f} 0.47(10 \%$ methanol/dichloromethane); $\mathrm{mp} 155-158{ }^{\circ} \mathrm{C}$. IR (KBr): $\widetilde{v} 1623.2,1727.5(\mathrm{C}=\mathrm{O}) \mathrm{cm}^{-1} .{ }^{1} \mathrm{H}$ NMR $\left(200 \mathrm{MHz}, \mathrm{CDCl}_{3}\right): \delta 0.88\left[\mathrm{t}, J=6.7 \mathrm{~Hz}, 3 \mathrm{H}, \mathrm{CH}_{3}\left(\mathrm{CH}_{2}\right)_{11}\right], 1.25-1.91\left[\mathrm{~m}, 30 \mathrm{H}, \mathrm{CH}_{3}\left(\mathrm{CH}_{2}\right)_{10} \mathrm{CH}_{2}\right.$, $\left.\left(\mathrm{CH}_{2}\right)_{5}\right], 2.67\left(\mathrm{dd}, J=8.17,16.9 \mathrm{~Hz}, 1 \mathrm{H}, \mathrm{CH}_{\mathrm{A}} \mathrm{CO}_{2} \mathrm{H}\right), 3.08-3.31\left(\mathrm{~m}, 3 \mathrm{H}, \mathrm{CH}_{\mathrm{B}} \mathrm{CO}_{2} \mathrm{H}, \mathrm{NCH}_{2}\right)$, $4.12(\mathrm{dd}, J=5.67,8.14 \mathrm{~Hz}, 1 \mathrm{H}, 2-\mathrm{CH})$. FAB-MS: $m / z(\%) 398(100)[\mathrm{M}+\mathrm{H}]^{+}$. Anal. Calcd for $\mathrm{C}_{22} \mathrm{H}_{39} \mathrm{NO}_{3} \mathrm{~S}$ (397.62): C, 66.45; H, 9.89; N, 3.52. Found: C, 66.44; H, 10.05; N, 3.48.

4-Dodecyl-3-oxo-1-thia-4-azaspiro[4.4]non-2-yl)-acetic acid (8c). Applying General Procedure B, laurylamine $\mathbf{1 b}(0.23 \mathrm{~mL}, 1.0 \mathrm{mmol})$, mercaptosuccinic acid 2c (450 $\mathrm{mg}$, $3.0 \mathrm{mmol})$ and cyclopentanone $3 \mathbf{b}(0.18 \mathrm{~mL}, 2.0 \mathrm{mmol})$ gave 8c $(275 \mathrm{mg}, 72 \%)$ as a gum; $\mathrm{R}_{f}$ 0.47 (10\% methanol/dichloromethane). IR (neat): $\widetilde{v} 1637.9,1729.8(\mathrm{C}=\mathrm{O}) \mathrm{cm}^{-1} .{ }^{1} \mathrm{H}$ NMR $(200$ $\left.\mathrm{MHz}, \mathrm{CDCl}_{3}\right): \delta 0.88\left[\mathrm{t}, J=6.66 \mathrm{~Hz}, 3 \mathrm{H}, \mathrm{CH}_{3}\left(\mathrm{CH}_{2}\right)_{11}\right], 1.25-1.99\left(\mathrm{~m}, 28 \mathrm{H}, \mathrm{CH}_{3}\left(\mathrm{CH}_{2}\right)_{10} \mathrm{CH}_{2}\right.$, $\left.\left(\mathrm{CH}_{2}\right)_{4}\right], 2.71\left(\mathrm{dd}, J=7.35,16.85 \mathrm{~Hz}, 1 \mathrm{H}, \mathrm{CH}_{\mathrm{A}} \mathrm{CO}_{2} \mathrm{H}\right), 3.01-3.33\left(\mathrm{~m}, 3 \mathrm{H}, \mathrm{CH}_{\mathrm{B}} \mathrm{CO}_{2} \mathrm{H}, \mathrm{CH}_{2}\right), 4.16$ $(\mathrm{dd}, J=6.95,13.75 \mathrm{~Hz}, 1 \mathrm{H}, 2-\mathrm{CH})$. FAB-MS: $m / z(\%) 384(100)[\mathrm{M}+\mathrm{H}]^{+}$. Anal. Calcd for $\mathrm{C}_{21} \mathrm{H}_{37} \mathrm{NO}_{3} \mathrm{~S}$ (383.59): C, 65.75; H, 9.72; N, 3.65. Found: C, 65.80; H, 9.76; N, 3.58.

4-Dodecyl-3-oxo-1-thia-4-azaspiro[4.6]undec-2-yl)-acetic acid (8d). Applying General Procedure B, laurylamine $\mathbf{1 b}(0.23 \mathrm{~mL}, 1.0 \mathrm{mmol})$, mercaptosuccinic acid 2c (450 $\mathrm{mg}$, $3.0 \mathrm{mmol})$ and cycloheptanone $3 \mathbf{c}(0.24 \mathrm{~mL}, 2.0 \mathrm{mmol})$ gave $8 \mathbf{d}(287 \mathrm{mg}, 70 \%)$ as a gum; $\mathrm{R}_{f}$ 0.48 (10\% methanol/dichloromethane). IR (neat): $\widetilde{v} 1639.6,1720.7(\mathrm{C}=\mathrm{O}) \mathrm{cm}^{-1} .{ }^{1} \mathrm{H}$ NMR $(200$ $\left.\mathrm{MHz}, \mathrm{CDCl}_{3}\right): \delta 0.88\left[\mathrm{t}, J=6.95 \mathrm{~Hz}, 3 \mathrm{H}, \mathrm{CH}_{3}\left(\mathrm{CH}_{2}\right)_{11}\right], 1.22-1.94\left[\mathrm{~m}, 32 \mathrm{H}, \mathrm{CH}_{3}\left(\mathrm{CH}_{2}\right)_{10} \mathrm{CH}_{2}\right.$, $\left.\left(\mathrm{CH}_{2}\right)_{6}\right], 2.63\left(\mathrm{dd}, J=8.08,15.79 \mathrm{~Hz}, 1 \mathrm{H}, \mathrm{CH}_{\mathrm{A}} \mathrm{CO}_{2} \mathrm{H}\right), 3.13-3.38\left(\mathrm{~m}, 3 \mathrm{H}, \mathrm{CH}_{\mathrm{B}} \mathrm{CO}_{2} \mathrm{H}, \mathrm{NCH}_{2}\right)$, $4.11(\mathrm{dd}, J=6.45,13.94 \mathrm{~Hz}, 1 \mathrm{H}, 2-\mathrm{CH})$. FAB-MS: $\mathrm{m} / \mathrm{z}(\%) 412(100)[\mathrm{M}+\mathrm{H}]^{+}$. Anal. Calcd for $\mathrm{C}_{23} \mathrm{H}_{41} \mathrm{NO}_{3} \mathrm{~S}$ (411.64): C, 67.11; H, 10.04; N, 3.40. Found: C, 67.15; H, 9.96; N, 3.46.

4-Dodecyl-3-oxo-1-thia-4azaspiro[4.7]dodec-2-yl)-acetic acid (8e). Applying General Procedure B, laurylamine $\mathbf{1 b}(0.23 \mathrm{~mL}, 1.0 \mathrm{mmol})$, mercaptosuccinic acid 2c (450 $\mathrm{mg}$, $3.0 \mathrm{mmol})$ and cyclooctanone $3 \mathbf{d}(0.26 \mathrm{~mL}, 2.0 \mathrm{mmol})$ gave $\mathbf{8 e}(323 \mathrm{mg}, 76 \%)$ as a gum; $\mathrm{R}_{f} 0.41$ (10\% methanol/dichloromethane). IR (neat): $\widetilde{v} 1671.7,1713.9(\mathrm{C}=\mathrm{O}) \mathrm{cm}^{-1} .{ }^{1} \mathrm{H} \mathrm{NMR}(200 \mathrm{MHz}$, $\left.\mathrm{CDCl}_{3}\right): \delta 0.88\left[\mathrm{t}, J=6.52 \mathrm{~Hz}, 3 \mathrm{H}, \mathrm{CH}_{3}\left(\mathrm{CH}_{2}\right)_{11}\right], 1.20-1.93\left[\mathrm{~m}, 34 \mathrm{H}, \mathrm{CH}_{3}\left(\mathrm{CH}_{2}\right)_{10} \mathrm{CH}_{2},\left(\mathrm{CH}_{2}\right)_{7}\right]$, 2.69 (dd, $\left.J=6.65,16.51 \mathrm{~Hz}, 1 \mathrm{H}, \mathrm{CH}_{\mathrm{A}} \mathrm{CO}_{2} \mathrm{H}\right), 3.02-3.34\left(\mathrm{~m}, 3 \mathrm{H}, \mathrm{CH}_{\mathrm{B}} \mathrm{CO}_{2} \mathrm{H}, \mathrm{NCH}_{2}\right), 4.13$ (dd, $J$ 
$=6.85,13.8 \mathrm{~Hz}, 1 \mathrm{H}, 2-\mathrm{CH})$. FAB-MS: $\mathrm{m} / \mathrm{z}(\%) 426(100)[\mathrm{M}+\mathrm{H}]^{+}$. Anal. Calcd for $\mathrm{C}_{24} \mathrm{H}_{43} \mathrm{NO}_{3} \mathrm{~S}$ (425.67): C, 67.72; H, 10.18; N, 3.29. Found: C, 67.74; H, 10.15; N, 3.25.

(8-tert-Butyl-4-dodecyl-3-oxo-1-thia-4-azaspiro[4.5]dec-2-yl)-acetic acid (9a). Applying General Procedure B, laurylamine $\mathbf{1 b}(0.23 \mathrm{~mL}, 1.0 \mathrm{mmol})$, mercaptosuccinic acid 2c (450 $\mathrm{mg}$, $3.0 \mathrm{mmol})$ and 4-tert-butylcyclohexanone 3e (308 $\mathrm{mg}, 2.0 \mathrm{mmol})$ gave 9a (312 $\mathrm{mg}, 69 \%)$ as a gum;. $\mathrm{R}_{f} 0.43$ (10\% methanol/dichloromethane). IR (neat): $\widetilde{v} 1639.0,1721.6(\mathrm{C}=\mathrm{O}) \mathrm{cm}^{-1} .{ }^{1} \mathrm{H}$ NMR $\left(200 \mathrm{MHz}, \mathrm{CDCl}_{3}\right): \delta$ 0.86-0.90 $\left[\mathrm{m}, 12 \mathrm{H}, \mathrm{CH}_{3}\left(\mathrm{CH}_{2}\right)_{11}, \mathrm{C}\left(\mathrm{CH}_{3}\right)_{3}\right], 1.26-1.96[\mathrm{~m}, 29 \mathrm{H}$, $\left.\mathrm{CH}_{3}\left(\mathrm{CH}_{2}\right)_{10} \mathrm{CH}_{2},\left(\mathrm{CH}_{2}\right)_{5}\right], 2.69\left(\mathrm{dd}, J=7.14,16.78 \mathrm{~Hz}, 1 \mathrm{H}, \mathrm{CH}_{\mathrm{A}} \mathrm{CO}_{2} \mathrm{H}\right), 3.10-3.31(\mathrm{~m}, 3 \mathrm{H}$, $\left.\mathrm{CH}_{\mathrm{B}} \mathrm{CO}_{2} \mathrm{H}, \mathrm{NCH}_{2}\right), 4.13(\mathrm{dd}, J=7.0,13.87 \mathrm{~Hz}, 1 \mathrm{H}, 2-\mathrm{CH})$. FAB-MS: $\mathrm{m} / \mathrm{z}$ (\%) 454 (100) $[\mathrm{M}+\mathrm{H}]^{+}$. Anal. Calcd for $\mathrm{C}_{26} \mathrm{H}_{46} \mathrm{~N}_{2} \mathrm{O}_{5} \mathrm{~S}$ (453.72): C, 68.83; H, 10.44; N, 3.09. Found: C, 68.91; H, 10.49; N, 3.17.

[8-(tert-Butoxycarbonyl)-4-dodecyl-3-oxo-1-thia-4,8-diazaspiro[4.5]dec-2-yl]acetic acid (9b). Applying General Procedure B, laurylamine $1 \mathbf{b}(0.23 \mathrm{~mL}, 1.0 \mathrm{mmol})$, mercaptosuccinic acid 2c (450 mg, $3.0 \mathrm{mmol})$ and $N$-Boc-piperidone $3 f(398 \mathrm{mg}, 2.0 \mathrm{mmol})$ gave $9 \mathbf{b}(378 \mathrm{mg}, 76 \%)$ as a gum; $\mathrm{R}_{f} 0.41$ (10\% methanol/dichloromethane). IR (neat): $\widetilde{v} 1641.2,1719.6(\mathrm{C}=\mathrm{O}) \mathrm{cm}^{-1} .{ }^{1} \mathrm{H}$ NMR $\left(200 \mathrm{MHz}, \mathrm{CDCl}_{3}\right): \delta 0.88\left[\mathrm{t}, J=6.75 \mathrm{~Hz}, 3 \mathrm{H}, \mathrm{CH}_{3}\left(\mathrm{CH}_{2}\right)_{11}\right], 1.26-2.09[\mathrm{~m}, 24 \mathrm{H}$, $\left.\mathrm{CH}_{3}\left(\mathrm{CH}_{2}\right)_{10} \mathrm{CH}_{2}, 6,10-\mathrm{CH}_{2}\right), 1.47$ [s, 9H, $\left.\mathrm{C}\left(\mathrm{CH}_{3}\right)_{3}\right], 2.71(\mathrm{dd}, J=7.97,17.07 \mathrm{~Hz}, 1 \mathrm{H}$, $\mathrm{CH}_{\mathrm{A}} \mathrm{CO}_{2} \mathrm{H}$ ), 2.87-3.29 (m, 5H, $\left.\mathrm{CH}_{\mathrm{B}} \mathrm{CO}_{2} \mathrm{H}, \mathrm{NCH}_{2}, 7-, 9-\mathrm{CH}_{2} \mathrm{~N}\right), 4.12-4.25$ (m, 3H, 2-CH, 7-, 9$\left.\mathrm{CH}_{2} \mathrm{~N}\right)$. FAB-MS: $\mathrm{m} / \mathrm{z}(\%) 499(100)[\mathrm{M}+\mathrm{H}]^{+}$. Anal. Calcd for $\mathrm{C}_{26} \mathrm{H}_{46} \mathrm{~N}_{2} \mathrm{O}_{5} \mathrm{~S}$ (498.72): C, 62.62; H, 9.30; N, 5.62. Found: C, 62.67; H, 9.35; N, 5.65.

2-(4-Dodecyl-3-oxo-1-thia-4,8-diazaspiro[4.5]dec-2-yl)acetic acid hydrochloride (10). Compound 9b (498 mg, $1 \mathrm{mmol}$ ) was kept in 20\% HCl/dioxane solution for $1 \mathrm{~h}$. The solventn was removed under reduced pressure, and the crude product was crystallized from dry diethyl ether to give product 10 (413 mg, 95\%) as a white hygroscopic solid; $\mathrm{R}_{f} 0.37(20 \%$ methanol/dichloromethane with 2 drops of acetic acid). IR (neat): $\widetilde{v} 1675.9,1743.1(\mathrm{C}=\mathrm{O}) \mathrm{cm}^{-1}$. ${ }^{1} \mathrm{H}$ NMR (200 MHz, CD $\left.{ }_{3} \mathrm{OD}\right): \delta 0.88\left[\mathrm{t}, J=6.70 \mathrm{~Hz}, 3 \mathrm{H}, \mathrm{CH}_{3}\left(\mathrm{CH}_{2}\right)_{11}\right], 1.27$ [br s, 20H, $\left.\mathrm{CH}_{3}\left(\mathrm{CH}_{2}\right)_{10} \mathrm{CH}_{2}\right], 1.95-2.11\left(\mathrm{~m}, 4 \mathrm{H}, 6,10-\mathrm{CH}_{2}\right), 2.69\left(\mathrm{dd}, J=8.02,16.99 \mathrm{~Hz}, 1 \mathrm{H}, \mathrm{CH}_{\mathrm{A}} \mathrm{CO}_{2} \mathrm{H}\right)$, 3.12-3.37 (m, 5H, $\mathrm{CH}_{\mathrm{B}} \mathrm{CO}_{2} \mathrm{H}, \mathrm{NCH}_{2}$, 7-, 9- $\left.\mathrm{CH}_{2} \mathrm{~N}\right), 4.19-4.37$ (m, 3H, 2-CH, 7-, 9- $\mathrm{CH}_{2} \mathrm{~N}$ ). FABMS: $m / z(\%) 399(100)[\mathrm{M}+\mathrm{H}]^{+}$. Anal. Calcd for $\mathrm{C}_{21} \mathrm{H}_{39} \mathrm{ClN}_{2} \mathrm{O}_{3} \mathrm{~S}$ (435.06): C, 57.97; H, 9.04; N, 6.44. Found: C, 58.10; H, 9.11; N, 6.56.

Synthesis of 8-substituted (4-dodecyl-3-oxo-1-thia-4,8-diazaspiro[4.5]dec-2-yl)acetic acid hydrochlorides (12a-c) General procedure C. To $9 \mathbf{b}(4.98 \mathrm{gm}, 10.0 \mathrm{mmol})$ was added a solution of diazomethane in ether (approx. $70-90 \mathrm{mmol}$ in $100 \mathrm{~mL}$ ether) at $0-5{ }^{\circ} \mathrm{C}$. The solvent was then evaporated. and the crude methyl ester was treated with a $20 \% \mathrm{HCl} /$ dioxane solution. Dioxane was evaporated at reduced pressure, and the crude salt was crystallized with dry diethyl ether. The crystalline material (449 $\mathrm{mg}, 1 \mathrm{mmol})$ was added to dry THF $(30 \mathrm{~mL})$ in a round bottom flask, and diisopropylethyl amine (DIEA) $(0.52 \mathrm{~mL}, 3.0 \mathrm{mmol})$, a Boc-protected amino acid (1.2 mmol) and 2-(1H-benzotriazol-1-yl)-1,1,3,3-tetramethyluronium hexafluorophosphate (HBTU) (455 mg, $1.2 \mathrm{mmol}$ ) were added. The reaction mixture was stirred at room temperature for $1 \mathrm{~h}$. After concentration under reduced pressure the residue was taken-up in ethyl acetate and 
washed with $5 \%$ citric acid solution $(2 \mathrm{x})$, with saturated sodium chloride solution, with $5 \%$ sodium carbonate solution $(2 \mathrm{x})$, and with saturated sodium chloride solution $(2 \mathrm{x})$. The ethyl acetate layer was dried over sodium sulfate and concentrated under reduced pressure to dryness. The crude product was purified by column chromatography on silica gel using methanol/dichloromethane as eluent. The purity of the final compounds was checked by tlc using methanol/dichloromethane (1:9) as solvent. The product was stirred with $\mathrm{LiOH} \mathrm{(48} \mathrm{mg}$, $2.0 \mathrm{mmol})$ in $\mathrm{THF} / \mathrm{methanol} /$ water $(7: 2: 1,20 \mathrm{~mL})$ for $1 \mathrm{~h}$ to give the lithium salt of corresponding acid, which was then acidified with citric acid solution and extracted with ethyl acetate. The organic layer was dried over sodium sulfate and evaporated under the reduced pressure to give the free acid derivative. The acid derivative was kept in $20 \% \mathrm{HCl} /$ dioxane $(30 \mathrm{~mL})$ for $1 \mathrm{~h}$; dioxane was removed under reduced pressure to give the crude product, which was crystallized from dry diethyl ether to give the hydrochloride salt $(\mathbf{1 2 a}-\mathbf{c} \cdot \mathrm{HCl})$. The purity of the final compounds was checked by tlc using methanol/dichloromethane (2:8) with two drops of acetic acid as solvent.

(4-Dodecyl-3-oxo-8-phenylalanyl-1-thia-4,8-diazaspiro[4.5]dec-2-yl)acetic acid hydrochloride (12a·HCl). Following General Procedure C, $9 \mathbf{b}(498 \mathrm{mg}, 1 \mathrm{mmol})$ and N-Boc-protected phenylalanine (318 $\mathrm{mg}, 1.2 \mathrm{mmol}$ ) formed 12a $\cdot \mathrm{HCl}(505 \mathrm{mg}, 87 \%)$ as a white hygroscopic solid; $\mathrm{R}_{f} 0.27$ (20\% methanol/dichloromethane with 2 drops of acetic acid). IR (KBr): $\widetilde{v}$ 1650.4, $1729.5(\mathrm{C}=\mathrm{O}) \mathrm{cm}^{-1} .{ }^{1} \mathrm{H}$ NMR $\left(200 \mathrm{MHz}, \mathrm{CD}_{3} \mathrm{OD}\right): \delta 0.87\left[\mathrm{t}, J=6.4 \mathrm{~Hz}, 3 \mathrm{H}, \mathrm{CH}_{3}\left(\mathrm{CH}_{2}\right)_{11}\right], 1.25-$ 2.57 (m, 24H, $\left.\mathrm{CH}_{3}\left(\mathrm{CH}_{2}\right)_{10} \mathrm{CH}_{2}, 6-, 10-\mathrm{CH}_{2}\right), 2.63-3.23$ (m, 8H, $\mathrm{CHCH}_{2} \mathrm{COOH}, 4-\mathrm{NCH}_{2}, \mathrm{CH}_{2} \mathrm{Ph}$, 7, 9- $\left.\mathrm{CH}_{2} \mathrm{~N}\right), 3.71-4.20(\mathrm{~m}, 2 \mathrm{H}, 2-\mathrm{CH}, \mathrm{NCH}), 4.63-4.94\left(\mathrm{~m}, 2 \mathrm{H}, 7,9-\mathrm{CH}_{2} \mathrm{~N}\right), 6.87-7.33(\mathrm{~m}, 5 \mathrm{H}$, $\mathrm{Ph})$. FAB-MS: $\mathrm{m} / \mathrm{z}(\%) 546(100)[\mathrm{M}+\mathrm{H}]^{+}$. Anal. Calcd for $\mathrm{C}_{30} \mathrm{H}_{48} \mathrm{ClN}_{3} \mathrm{O}_{4} \mathrm{~S}$ (582.24): C, 61.89; H, 8.31; N, 7.22. Found: C, 62.01; H, 8.43; N, 7.35.

(4-Dodecyl-8-isoleucyl-3-oxo-1-thia-4,8-diazaspiro[4.5]dec-2-yl)acetic acid hydrochloride (12b·HCl). Following General Procedure C, 9b (498 mg, $1 \mathrm{mmol}$ ) and N-Boc-protected isoleucine (277 mg, $1.2 \mathrm{mmol}$ ) formed $\mathbf{1 2 b} \cdot \mathrm{HCl}(465 \mathrm{mg}, 85 \%)$ as a white hygroscopic solid; $\mathrm{R}_{f}$ 0.27 (20\% methanol/dichloromethane with 2 drops of acetic acid). IR (KBr): $\widetilde{v} 1648.6,1726.6$

$(\mathrm{C}=\mathrm{O}) \mathrm{cm}^{-1} .{ }^{1} \mathrm{H}$ NMR $\left(200 \mathrm{MHz}, \mathrm{CD}_{3} \mathrm{OD}\right): \delta 0.87 \quad\left[\mathrm{t}, J=6.54 \mathrm{~Hz}, 9 \mathrm{H}, \mathrm{CH}_{3}\left(\mathrm{CH}_{2}\right)_{11}\right.$, $\left.\mathrm{CH}_{3} \mathrm{CH}_{2} \mathrm{CHCH}_{3}\right], 1.25-2.57$ [m, 26H, $\mathrm{CH}_{3}\left(\mathrm{CH}_{2}\right)_{10} \mathrm{CH}_{2}, \mathrm{CH}_{3} \mathrm{CH}_{2} \mathrm{CHCH}_{3}, 6-, 10-\mathrm{CH}_{2}$ ], 2.67-3.44 $\left(\mathrm{m}, 6 \mathrm{H}, \mathrm{CHCH}_{2} \mathrm{CO}_{2} \mathrm{H}, 4-\mathrm{NCH}_{2}, 7-, 9-\mathrm{CH}_{2} \mathrm{~N}\right), 4.09-4.20(\mathrm{~m}, 2 \mathrm{H}, 2-\mathrm{CH}, \mathrm{NCH}), 4.42-4.79$ (m, $\left.2 \mathrm{H}, 7-, 9-\mathrm{CH}_{2} \mathrm{~N}\right)$. FAB-MS: $\mathrm{m} / \mathrm{z}(\%) 512(100)[\mathrm{M}+\mathrm{H}]^{+}$. Anal. Calcd for $\mathrm{C}_{27} \mathrm{H}_{50} \mathrm{ClN}_{3} \mathrm{O}_{4} \mathrm{~S}$ (548.22): C, 59.15; H, 9.19; N, 7.66. Found: C, 59.29; H, 9.28; N, 7.74.

(4-Dodecyl-8-lysyl-3-oxo-1-thia-4,8-diazaspiro[4.5]dec-2-yl)acetic acid hydrochloride (12c·HCl). Following General Procedure C, 9b (498 mg, $1 \mathrm{mmol})$ and bis-Boc-protected lysine (415 mg, $1.2 \mathrm{mmol})$ formed 12c $\cdot \mathrm{HCl}(472 \mathrm{mg}, 79 \%)$ as a brown hygroscopic solid; $\mathrm{R}_{f} 0.15(20 \%$ methanol/dichloromethane with 2 drops of acetic acid). IR $(\mathrm{KBr}): \widetilde{v} 1653.8,1725.2(\mathrm{C}=\mathrm{O}) \mathrm{cm}^{-1}$. ${ }^{1} \mathrm{H}$ NMR $\left(200 \mathrm{MHz}, \mathrm{CD}_{3} \mathrm{OD}\right): \delta 0.88\left[\mathrm{t}, J=6.58 \mathrm{~Hz}, 3 \mathrm{H}, \mathrm{CH}_{3}\left(\mathrm{CH}_{2}\right)_{11}\right], 1.22-2.26[\mathrm{~m}, 28 \mathrm{H}$, $\left.\mathrm{CH}_{3}\left(\mathrm{CH}_{2}\right)_{10} \mathrm{CH}_{2}, \mathrm{CHCH}_{2} \mathrm{CH}_{2} \mathrm{CH}_{2} \mathrm{CH}_{2} \mathrm{NH}_{2}, 6,10-\mathrm{CH}_{2}\right], 2.62-3.38$ (m, 8H, $\mathrm{CHCH}_{2} \mathrm{CO}_{2} \mathrm{H}, 4-$ $\left.\mathrm{NCH}_{2}, \mathrm{CHCH}_{2} \mathrm{CH}_{2} \mathrm{CH}_{2} \mathrm{CH}_{2} \mathrm{~N}, 7-, 9-\mathrm{CH}_{2} \mathrm{~N}\right), 4.0-4.18$ (m, 2H, 2-CH, $\mathrm{NCH}$ ), 4.61-4.78 (m, 2H, 7, 
9- $\mathrm{CH}_{2} \mathrm{~N}$ ). FAB-MS: m/z (\%) 527 (100) $[\mathrm{M}+\mathrm{H}]^{+}$. Anal. Calcd for $\mathrm{C}_{27} \mathrm{H}_{52} \mathrm{Cl}_{2} \mathrm{~N}_{4} \mathrm{O}_{4} \mathrm{~S}$ (599.70): C, 54.08; H, 8.74; N, 9.34. Found: C, 54.18; H, 8.81; N, 9.46.

\section{3-Amino-4-[2-(carboxymethyl)-4-dodecyl-3-oxo-1-thia-4,8-diazaspiro[4.5]dec-8-yl]-4-oxo-}

butanoic acid hydrochloride (12d·HCl). To $9 \mathbf{b}(498 \mathrm{mg}, 1.0 \mathrm{mmol})$ was treated following General Procedure $\mathrm{C}$ until the preparation of the corresponding N-deprotected acid derivative. in In a round bottom flask containing $30 \mathrm{~mL}$ methanol were added the acid derivative, a pinch of $\mathrm{Pd} / \mathrm{C}$, and through the reaction mixture with a stream of hydrogen gas was bubbled for $2 \mathrm{~h}$. The solution was filtered through a sintered funnel with a celite pad. The filtrate was concentrated under reduced pressure to give the corresponding O-debenzylated product, which was kept in $20 \% \mathrm{HCl} /$ dioxane $(30 \mathrm{~mL})$ for $1 \mathrm{~h}$. The solvent was removed under reduced pressure to give the crude salt, which was crystallized with dry diethyl ether to give $\mathbf{1 2 d} \cdot \mathrm{HCl}(411 \mathrm{mg}, 75 \%)$ as a white hygroscopic solid; $\mathrm{R}_{f} 0.17$ (20\% methanol/dichloromethane with 2 drops of acetic acid). IR (KBr): $\widetilde{v} 1635.9,1721.0(\mathrm{C}=\mathrm{O}) \mathrm{cm}^{-1} .{ }^{1} \mathrm{H}$ NMR $\left(200 \mathrm{MHz}, \mathrm{CD}_{3} \mathrm{OD}\right): \delta 0.87[\mathrm{t}, J=6.66 \mathrm{~Hz}, 3 \mathrm{H}$, $\left.\mathrm{CH}_{3}\left(\mathrm{CH}_{2}\right)_{11}\right], \quad 1.25-2.59 \quad\left[\mathrm{~m}, \quad 24 \mathrm{H}, \quad \mathrm{CH}_{3}\left(\mathrm{CH}_{2}\right)_{10} \mathrm{CH}_{2}, \quad 6-, \quad 10-\mathrm{CH}_{2}\right), \quad 2.65-3.48$ (m, 6H, $\left.\mathrm{CHCH}_{2} \mathrm{CO}_{2} \mathrm{H}, \mathrm{NCHCH}_{2} \mathrm{CO}_{2} \mathrm{H}, 4-\mathrm{NCH}_{2}, 7-, 9-\mathrm{CH}_{2} \mathrm{~N}\right), 4.05-4.22(\mathrm{~m}, 2 \mathrm{H}, 2-\mathrm{CH}, \mathrm{NCH}), 4.45-$ $4.81\left(\mathrm{~m}, 2 \mathrm{H}, \quad 7-, 9-\mathrm{CH}_{2} \mathrm{~N}\right)$. FAB-MS: $\mathrm{m} / \mathrm{z}(\%) 514$ (100) $[\mathrm{M}+\mathrm{H}]^{+}$. Anal. Calcd for $\mathrm{C}_{25} \mathrm{H}_{44} \mathrm{ClN}_{3} \mathrm{O}_{6} \mathrm{~S}$ (550.15): C, 54.58; H, 8.06; N, 7.64. Found: C, 54.70; H, 8.21; N, 7.73.

\section{Acknowledgements}

One of us TS thanks the CSIR, New Delhi for financial assistance. We are also thankful to the Director of CDRI for the support and the SIAF for the spectral data. CDRI communication no. 6629.

\section{References}

1. Narain, J. P., Tripathy, S. P., Pontali, E., In Tuberculosis epidemiology and control Narain, J. P. Ed;; WHO Document \# SEA/TB/248, 2002; Ch. 6, p 83.

2. Babaoglu, K.; Page, M. A.; Jones, V. C.; McNeil, M. R.; Dong, C.; Naismith, J. H.; Lee, R. E. Bioorg. Med. Chem. Lett. 2003, 13, 3227.

3. Srivastava, T.; Haq, W.; Katti, S. B. Tetrahedron 2002, 58, 7619.

4. Homes, C. P.; Chinn, J. P.; Look, G. C.; Gordon, E. M.; Gallop, M. A. J. Org. Chem. 1995, 60, 7328.

5. Collins, L.; Franzblau, S. G. Antimicrob. Agents Chemother. 1997, 41, 1004. 